\title{
Preoperative serum CA125 level and age at diagnosis: An effective prognosis prediction tool for patients with early-stage endometrial cancer
}

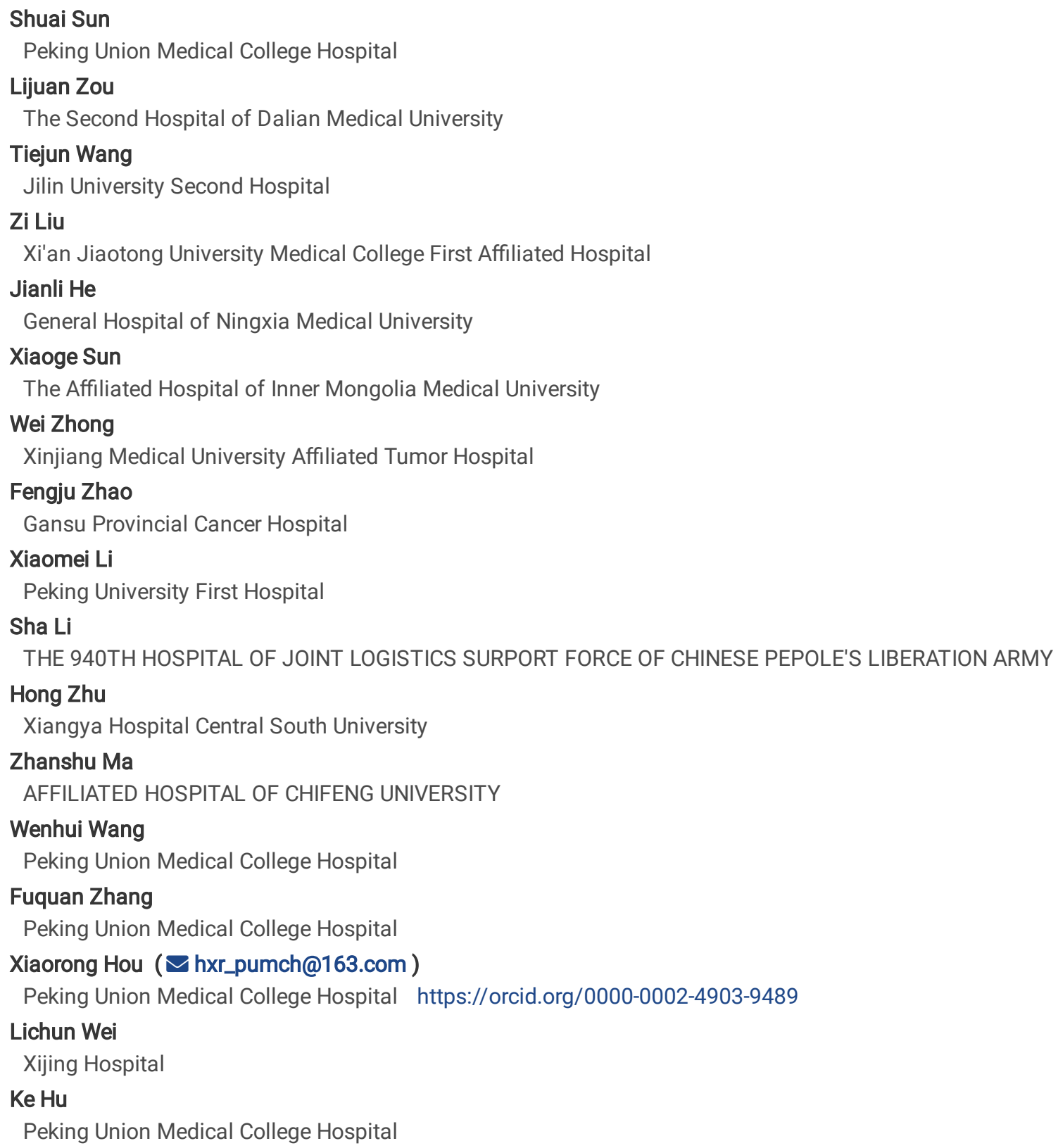

\section{Research Article}

Keywords: early-stage endometrial cancer, preoperative serum CA125, age at diagnosis

Posted Date: January 27th, 2022

DOI: https://doi.org/10.21203/rs.3.rs-1287680/v1

License: (c) (i) This work is licensed under a Creative Commons Attribution 4.0 International License. Read Full License 


\section{Abstract \\ Objective}

To investigate the combined predictive value of the preoperative serum cancer antigen 125 (CA125) level and age at diagnosis among patients with early-stage endometrial cancer(EC) after initial treatment.

\section{Methods}

We retrospectively analyzed data from patients with early-stage EC from January 1999 to December 2015 in multiple institutions in China. All patients received primary hysterectomy \pm bilateral salpingo-oopho rectomy and adjuvant radiotherapy for pathology-confirmed stage I and II EC(FIGO 2009 staging). In total, 447 patients with complete data on preoperative serum CA125 levels were included. All patients were divided into low-risk, intermediate-risk, high-intermediate-risk and high-risk groups according to the ESMO-ESGO-ESTRO risk classification. Binary logistic regression was used to calculate the predictive probability of 5-year overall survival (OS) for each patient, and the receiver operating characteristic (ROC) curve was used to calculate the sensitivity and specificity of variables, as well as the combined predictive value of CA125 and age.

\section{Results}

The median follow-up time was 59 months (3-201 months). The 5-year OS and disease-free survival (DFS) rates were $94.4 \%$ and $89.1 \%$, respectively. Multivariate analysis showed that the preoperative CA125 level and age at diagnosis were independent prognostic factors for 5year OS. The best cutoff value forage at diagnosis was 61 years. The cutoff values of CA125 for patients aged $\leq 61$ years and > 61 years were $37.7 \mathrm{U} / \mathrm{mL}$ and $39.35 \mathrm{U} / \mathrm{mL}$, respectively. Patients aged $>61$ years with $\mathrm{CA} 125>39.35 \mathrm{U} / \mathrm{ml}$ and patients aged $\leq 61$ years with CA125 > $37.7 \mathrm{U} / \mathrm{ml}$ were classified as theCA125-positive group; the remaining patients constituted the CA125-negative group. The area under the curve (AUC) for CA125 combined with age at diagnosis was 0.692 , and the corresponding sensitivity and specificity were $68.2 \%$ and $68.2 \%$ $(P<0.002)$, respectively, which were significantly better than the corresponding values for CA125 or age alone.

\section{Conclusions}

Although preoperative CA125 had limited sensitivity in predicting the prognosis for early-stage EC after initial treatment, it remains a useful serum marker for risk assessment of early-stage EC. Combining CA125 with age may increase its predictive sensitivity.

\section{Introduction}

Endometrial cancer (EC) is the most common gynecological malignant tumor, and its incidence is increasing. Although most patients are diagnosed early and cured by hysterectomy, $15-20 \%$ of patients experience recurrence without locally advanced or metastatic disease[1]. Therefore, identifying adverse factors related to recurrence and metastasis from clinicopathological information for patients with early-stage EC and providing appropriate treatment is especially beneficial for improving overall survival (OS). Serum biomarkers are of great value for the early detection of primary and recurrent diseases, monitoring responses to adjuvant therapy, preoperative selection of high-risk patients, and more personalized treatment. Cancer antigen 125 (CA125) levels have been shown to be associated with a number of clinicopathological factors in EC. However, most of the current studies on CA125 in EC are focused on the prediction of advanced EC[2-5], and the literature on the predictive role of CA125 in early-stage EC is limited. This study aimed to analyze the predictive value of preoperative serum CA125 combined with clinicopathological factors in patients with early-stage EC after initial treatment.

\section{Methods And Materials}

1. Ethics Approval and Informed Consent

This retrospective study was approved by the Ethics Review Committee of Peking Union Medical College Hospital, Chinese Academy of Medical Sciences [Protocol number S-K139]. The clinical trial ID of the study is ChiCTR-PRC-17010712. Evaluation of all data met the requirements of the Helsinki Declaration.

2. Materials and Evaluation 
After obtaining Institutional Review Board approval, we retrospectively reviewed the medical records in our multi-institutional EC database. A total of 1268 patients with early-stage EC treated at 13 grade A tertiary hospitals in China between January 1999 and December 2015 were included. Preoperative serum CA125 levels were recorded for 447 of the 1268 patients (Figure 1). All patients underwent primary hysterectomy \pm bilateral salpingo-oophorectomy, had confirmed FIG02009 stage I or stage II EC and had a World Health Organization performance score between 0 and2. All patients received postoperative adjuvant radiotherapy and were followed up for at least 3 months. Risk classification was carried out according to ESMO-ESGO-ESTRO risk classification. Treatment-related late toxicity was evaluated according to the Radiation Therapy Oncology Group(RTOG) criteria. The electrochemiluminescence method of Roche Cobas was used to detect CA125. The normal range of CA125 was $\leq 35 \mathrm{u} / \mathrm{ml}$.

Figure 1Flow chart of patient enrollment

\section{Radiotherapy Modalities}

All patients received postoperative pelvic external beam radiotherapy (EBRT) \pm vaginal brachytherapy (VBT) or VBT alone. The target volume of EBRT included the vaginal stump and upper 1/2 of the vagina, parauterine, presacral, obturator, internal and external iliac and common iliac lymphatic drainage areas. Conventional four-field "box" radiotherapy (CRT), three-dimensional conformal radiotherapy (3DCRT) and intensity-modulated radiotherapy (IMRT) were used in external beam irradiation. VBT was performed by irradiating the vaginal stump and upper $1 / 2$ of the vagina with single-channel or multichannel applicators, and two- and three-dimensional HDR brachytherapy plans were used for brachytherapy.

\section{Follow-up}

All patients had complete clinical, pathological, and follow-up information. Patients were assessed every 3-6 months for the first 2 years after radiotherapy, every 6-12 months during the following three years, and then annually. The prognostic factors analyzed included age at diagnosis, preoperative serum CA125 levels, surgical type, pathological type, grade, myometrial invasion (MI), lymphatic vascular space invasion (LVSI), lower uterine segmental invasion (LUSI), cervical stromal invasion(CSI), FIGO 2009 stage, ESMO-ESGO-ESTRO risk classification, time interval between surgery and radiotherapy, radiotherapy mode, and use of chemotherapy.

The primary endpoint was 5-year OS.The secondary endpoint was 5-year disease-free survival (DFS). The primary endpoint was defined as the time from the operation to death from any cause or the last follow-up. The secondary endpoint was defined as the time from the operation to disease recurrence or metastasis or the last follow-up. After the initial treatment, new lesions in the pelvic area were defined as local regional recurrence (including vaginal recurrence), and new lesions beyond the pelvic area were defined as distant metastasis.

\section{Statistical Analysis Methods}

The Kaplan-Meier method was used to calculate OS and DFS. Cox proportional hazard regression was used for multivariate analysis. Binary logistic regression was used to calculate the predictive probability of 5-year OS for each patient, and the ROC curve was used to calculate the sensitivity and specificity of the variables, as well as the combined predictive value of CA125 and age. All analyses were performed using IBM SPSS Statistics for Windows version 19.0, and $\mathrm{P}<0.05$ was considered statistically significant.

\section{Results}

1区Clinical characteristics

The median follow-up time was 59 months (3-201 months). Four hundred seven patients(91.1\%) were staged as I (FIGO2009), and forty patients(8.9\%) were staged as II. A total of 313 patients (70.0\%) underwent completely staged surgery. Among the 447 patients, $51.7 \%$ (231 cases) had deep MI, and 64\% (286 cases) had grade 2-3 MI. According to the ESMO-ESGO-ESTRO risk classification, there were $16.8 \%$ (75 cases) in the high-intermediate-risk group and $21.5 \%$ (96 cases) in the high-risk group. The median time interval between surgery and radiotherapy was 38 days (14-174 days), and the cutoff value of the time interval between surgery and radiotherapy for OS and DFS was 49.5 days. The median number of lymph node dissections was 18(range 1-65). In patients receiving EBRT, 33.6\% received CRT, $14.3 \%$ received 3DCRT, and $52.1 \%$ received IMRT. The dose range to EBRT was $40-50.4$ Gy in $25-28$ fractions. When VBT was used as a boost to EBRT, the dose to the vaginal mucosa was 8-25 Gy in 2-5 fractions. For postoperative VBT alone, the dose to the vaginal mucosa was $25-$ 40 Gy in 5-8 fractions. Ninety-five patients (21.3\%) underwent chemotherapy. The clinical characteristics and initial treatment of the patients are shown in Table 1.

2. Preoperative serum CA125 level and age at diagnosis 
Serum CA125 levels were negatively correlated with age $(P=0.001)$. The median preoperative serum CA125 level and age at diagnosis for 447 patients were $20.6 \mathrm{U} / \mathrm{ml}(1.9-863.2 \mathrm{U} / \mathrm{ml})$ and 57 years (23-86 years), respectively. The best cutoff value of age at diagnosis was 61 years $(A U C=0.654$, sensitivity $=54.5 \%$, specificity $=76.5 \%)$.

According to its cutoff value, age was divided into two groups: $\leq 61$ years and $>61$ years. For patients aged $\leq 61$ years, the median CA125value was $22.3 \mathrm{U} / \mathrm{ml}(1.9-863.2 \mathrm{U} / \mathrm{ml})$, and the cutoff value was $37.7 \mathrm{U} / \mathrm{ml}$ (AUC 0.535, sensitivity $=0.4$, specificity $=0.751)$. For patients aged $>61$ years, the median CA125 value was $19.48 \mathrm{U} / \mathrm{ml}(3.36-337.5 \mathrm{U} / \mathrm{ml})$, and the cutoff value was $39.35 \mathrm{U} / \mathrm{ml}(\mathrm{AUC} 0.609$, sensitivity $=0.417$, specificity $=0.840$ ). Patients aged $>61$ years with CA125 $>39.35 \mathrm{U} / \mathrm{ml}$ and patients aged $\leq 61$ yearswith $\mathrm{CA} 125>37.7 \mathrm{U} / \mathrm{ml}$ were classified as the CA125-positive group. In contrast, patients aged $>61$ years with CA125 $<39.35 \mathrm{U} / \mathrm{ml}$ and patients aged $\leq 61 \mathrm{yearswith}$ CA125 < $37.7 \mathrm{U} / \mathrm{ml}$ were classified as theCA125-negative group. Finally, CA125 was statistically analyzed in the form of a positive group and a negative group.

\section{Survival analysis}

The 5-year OS and DFS rates were $94.4 \%$ and 89.1\%, respectively. Among all 447 patients, 22 patients died, among whom 14 died of EC, 7 died of cardiovascular comorbidities and 1 died of secondary primary cancer. Of all 447 patients, 32 had recurrence or metastasis after initial treatment. In the first disease progression, 8 patients had local regional recurrence, 5 patients had both distant metastasis and local regional recurrence, and 19 patients had distant metastasis. The sites of metastasis included the peritoneum (peritoneal dissemination), lung, liver, bone, brain, adrenal gland and lymph node.

4. Analysis of prognostic factors

In the univariate analysis, preoperative serum CA125, surgical type and time interval between surgery and radiotherapy were significant for 5year OS. Age, surgical type, LVSI and time interval between surgery and radiotherapy were significant for 5-year DFS. As shown in Table 1.

Table 1 Clinical characteristics of 447 patients and univariate analysis for OS and DFS 


\begin{tabular}{|c|c|c|c|c|}
\hline Variable & & No. & $P$ for OS & P for DFS \\
\hline \multirow[t]{2}{*}{ Age } & $\leq 61 y$ & $335(74.9 \%)$ & \multirow[t]{2}{*}{0.001} & \multirow[t]{2}{*}{0.002} \\
\hline & $>61 y$ & $112(25.1 \%)$ & & \\
\hline \multirow[t]{2}{*}{ Preoperative serum CA125 } & Positive & $341(76.3 \%)$ & \multirow[t]{2}{*}{0.033} & \multirow[t]{2}{*}{0.154} \\
\hline & Negative & $106(23.7 \%)$ & & \\
\hline \multirow[t]{2}{*}{ Surgical type } & Completely surgically staged & $313(70.0 \%)$ & \multirow[t]{2}{*}{0.036} & \multirow[t]{2}{*}{0.095} \\
\hline & Incompletely surgically staged & $134(30.0 \%)$ & & \\
\hline \multirow[t]{2}{*}{ Pathology } & Endometrioid carcinoma & $416(93.1 \%)$ & \multirow[t]{2}{*}{0.673} & \multirow[t]{2}{*}{0.614} \\
\hline & Non-endometrioid carcinoma & $31(6.9 \%)$ & & \\
\hline \multirow[t]{3}{*}{ Grade $^{a}$} & Grade1 & $161(36.0 \%)$ & \multirow[t]{3}{*}{0.314} & \multirow[t]{3}{*}{0.321} \\
\hline & Grade2 & $187(41.8 \%)$ & & \\
\hline & Grade3 & $99(22.2 \%)$ & & \\
\hline \multirow[t]{2}{*}{ MI } & $\geq 1 / 2$ & $216(48.3 \%)$ & \multirow[t]{2}{*}{0.306} & \multirow[t]{2}{*}{0.374} \\
\hline & $<1 / 2$ & $231(51.7 \%)$ & & \\
\hline \multirow[t]{2}{*}{ LVSI } & Positive & $68(15.2 \%)$ & \multirow[t]{2}{*}{0.300} & \multirow[t]{2}{*}{0.008} \\
\hline & Negative & $379(84.8 \% \rrbracket$ & & \\
\hline \multirow[t]{2}{*}{ LUSI } & Yes & $171(38.3 \%)$ & \multirow[t]{2}{*}{0.681} & \multirow[t]{2}{*}{0.454} \\
\hline & No & $276(61.7 \%)$ & & \\
\hline \multirow[t]{2}{*}{ CSI } & Yes & $39(8.7 \%)$ & \multirow[t]{2}{*}{0.537} & \multirow[t]{2}{*}{0.109} \\
\hline & No & $408(91.3 \%)$ & & \\
\hline \multirow[t]{3}{*}{ FIG02009stage } & la & $213(47.7 \%)$ & \multirow[t]{3}{*}{0.495} & \multirow[t]{3}{*}{0.252} \\
\hline & $\mathrm{lb}$ & $194(43.4 \%)$ & & \\
\hline & II & $40(8.9 \%)$ & & \\
\hline ESMO-ESGO-ESTRO & Low risk & $136(30.4 \%)$ & 0.887 & 0.129 \\
\hline Risk classification & Intermediate risk & $140(31.3 \%)$ & & \\
\hline & High-intermediate risk & $75(16.8 \%)$ & & \\
\hline & High risk & $96(21.5 \%)$ & & \\
\hline Time interval between $S$ and $R$ & $\leq 49$ days & $314(70.2 \%)$ & 0.019 & 0.023 \\
\hline & $>49$ days & $133(29.8 \%)$ & & \\
\hline Radiotherapy mode & EBRT alone & $20(4.5 \%)$ & 0.150 & 0.517 \\
\hline & VBT alone & $295(66.0 \%)$ & & \\
\hline & $\mathrm{EBRT}+\mathrm{VBT}$ & $132(29.5 \%)$ & & \\
\hline Chemotherapy & Yes & $95(21.3 \%)$ & 0.601 & 0.583 \\
\hline & No & $352(78.7 \%)$ & & \\
\hline
\end{tabular}

aEndometrioid cancers were designated as grade 1, 2, or 3. All poorly differentiated cancers, uterine papillary serous cancers, and clear cell cancers were designated as grade 3. LUSI=lower uterine segment invasion, LVSI=lymphatic vascular space invasion, EBRT= external beam radiotherapy, $\mathrm{VBT}=$ vaginal brachytherapy, $\mathrm{MI}=$ myometrial invasion, $\mathrm{CSI}=$ cervical stromal invasion. $\mathrm{S}=$ surgery, $\mathrm{R}=$ radiotherapy

The results of the multivariate analysis of all 447 patients for OS showed that older age and CA125 positivity were independent prognostic factors for OS (see Table 2). The nomogram is shown in Figure 2. In the multivariate analysis for DFS, age, complete surgical stage, LVSI 
and time interval between surgery and radiotherapy were independent prognostic factors, while CA125 was not statistically significant in the univariate and multivariate analyses, as shown in Table 3.

Table 2 Results of the multivariate analysis of all 447 patients for OS

\begin{tabular}{|lllll|}
\hline Variable & HR & $\mathbf{P}$ & $\mathbf{9 5 \%} \mathrm{Cl}$ \\
\cline { 4 - 5 } & & & $\mathbf{2 . 5 \%}$ & $\mathbf{9 7 . 5 \%}$ \\
\hline Age & 4.33 & 0.0007 & 1.858 & 10.082 \\
\hline CA125 & 2.86 & 0.0162 & 1.215 & 6.732 \\
\hline
\end{tabular}

OS=Overall survival, $\mathrm{HR}=$ hazard ratio, $\mathrm{Cl}=$ confidence interval.

Age 0: $\leq 61 y, 1:>61 y, C A 125$ 0: negative, 1: positive.

Figure 2 Nomogram for 5y OS

Table 3 Results of the multivariate analysis of DFS in all 447 patients

\begin{tabular}{|lllll|}
\hline Variable & HR & P & 95\% Cl & \\
\cline { 4 - 5 } & & & $\mathbf{2 . 5 \%}$ & $\mathbf{9 7 . 5 \%}$ \\
\hline Age & 1.04 & 0.0117 & 1.010 & 1.078 \\
\hline Surgical type & 0.48 & 0.0329 & 0.2449 & 0.942 \\
\hline LVSI & 2.60 & 0.0065 & 1.3057 & 5.167 \\
\hline Interval & 1.01 & 0.0200 & 1.002 & 1.021 \\
\hline
\end{tabular}

We further analyzed the relationship between CA125 and clinical parameters. The clinical characteristics of each group are shown in Table 5.We found that there were more patients with deep MIs, with LUSI(+), with FIGO 2009 stage Ib and II, and receiving EBRT and chemotherapy $(P<0.05)$ in the CA125-positive group. The survival function is shown in Figure 3. There was a significant difference between the two groups. The 5-year OS of the patients in the CA125-positive group was 90.5\%, and the 5-year OS of the patients in the CA125-negative group was 95.5\% (P=0.033).

The 5-year OS rates among patients aged $\leq 61$ years and those aged $>61$ years were $96.2 \%$ and $88.8 \%$, respectively $(P=0.001)$. The survival function is shown in Figure 3.

Figure 3 Survival function of CA125(3-a) and age(3-b) alone and in combination(3-c) to predict 5-year OS

Finally, the relationship between CA125 combined with age and other clinical features was analyzed. The AUC of CA125 combined with age at diagnosis was 0.692 (sensitivity $=68.2 \%$, specificity $=68.2 \%, P<0.002$ ), which was significantly better than the predictive value of $C A 125$ or age alone, as shown in Table 4.

Table 4 Value of CA125 and age alone or in combination in predicting OS in early-stage EC

\begin{tabular}{|lllll|}
\hline & AUC & Sensitivity (\%) & Specificity (\%) & $P$-value \\
\hline CA125 combined with age & 0.692 & 0.682 & 0.682 & 0.002 \\
\hline CA125 alone & 0.590 & 0.409 & 0.772 & 0.152 \\
\hline Age alone & 0.654 & 0.545 & 0.765 & 0.015 \\
\hline
\end{tabular}

Therefore, patients were divided into 4 groups according to age and CA125: age $\leq 61$ years and CA125 < $37.7 \mathrm{U} / \mathrm{ml}$; age $\leq 61$ years and CA125 $>37.7 \mathrm{U} / \mathrm{ml}$; age $>61$ years and CA $125<39.35 \mathrm{U} / \mathrm{ml}$; and age >61 years and CA125 > 39.35. Inthe analysis of the clinicopathological factors of these four groups of patients, the proportion of complete surgical staging and stage la decreased gradually, and that of $\mathrm{MI}$ increased gradually (see Table 5). The 5-year OS rates of the four groups were 96.9\%, 93.6\%, 91.4\% and 75.6\%, respectively ( $P=0.000)($ see Figure 3 ).

Table 5The baseline characteristics of the447 patients with EC stratified by CA125 and age 


\begin{tabular}{|c|c|c|c|c|c|c|c|c|c|}
\hline \multicolumn{2}{|l|}{ Characteristic } & \multicolumn{2}{|c|}{ CA125groups } & \multirow[t]{3}{*}{$\mathbf{P}$} & \multicolumn{4}{|c|}{ Age and CA125 combined groups } & \multirow[t]{3}{*}{$\mathbf{P}$} \\
\hline & & \multirow{2}{*}{$\begin{array}{l}\text { Negative } \\
341(\%)\end{array}$} & \multirow{2}{*}{$\begin{array}{l}\text { Positive } \\
106(\%)\end{array}$} & & \multirow{2}{*}{$\begin{array}{l}1 \\
250(\%)\end{array}$} & \multirow{2}{*}{$\begin{array}{l}2 \\
85(\%)\end{array}$} & \multirow[t]{2}{*}{3} & \multirow[t]{2}{*}{4} & \\
\hline & & & & & & & & & \\
\hline \multirow[t]{2}{*}{ Age } & $\leq 61 y$ & $250(73.5 \%)$ & $85(80.2 \%)$ & \multirow[t]{2}{*}{0.161} & / & / & I & / & \multirow[t]{2}{*}{ / } \\
\hline & $>61 y$ & $91(26.7 \%)$ & $21(19.8 \%)$ & & / & / & / & / & \\
\hline \multicolumn{2}{|c|}{ Endometrioid carcinoma } & $318(93.3 \%)$ & $98(92.5 \%)$ & 0.827 & $234(93.6 \%)$ & $79(92.9 \%)$ & $84(92.3 \%)$ & 19(90.5) & 0.938 \\
\hline Surgical type & $\begin{array}{l}\text { Completely } \\
\text { surgically } \\
\text { staged }\end{array}$ & $242(71.0 \%)$ & $71(67.0 \%)$ & 0.467 & $186(74.4 \%)$ & $61(71.8 \%)$ & $56(61.5 \%)$ & $10(47.6 \%)$ & 0.014 \\
\hline \multirow[t]{3}{*}{ Grade } & $\mathrm{G} 1$ & $124(36.4 \%)$ & $37(34.9 \%)$ & \multirow[t]{3}{*}{0.963} & $93(37.2 \%)$ & $30(35.3 \%)$ & $31(34.1 \%)$ & $7(33.3 \%)$ & \multirow[t]{3}{*}{0.997} \\
\hline & G2 & $142(41.6 \%)$ & $45(42.5 \%)$ & & $104(41.6 \%)$ & $36(42.4 \%)$ & $38(41.8 \%)$ & $9(42.9 \%)$ & \\
\hline & G3 & $75(22.0 \%)$ & $24(22.6 \%)$ & & $53(21.2 \%)$ & $19(22.4 \%)$ & $22(24.2 \%)$ & $5(23.8 \%)$ & \\
\hline \multirow[t]{2}{*}{$\mathrm{Ml}$} & $\geq 1 / 2$ & $156(45.7 \%)$ & $60(56.6 \%)$ & \multirow[t]{2}{*}{0.049} & $100(40.0 \%)$ & $45(52.9 \%)$ & $56(61.5 \%)$ & $15(71.4 \%)$ & \multirow[t]{2}{*}{0.000} \\
\hline & $<1 / 2$ & $185(54.3 \%)$ & $46(43.4 \%)$ & & $150(60.0 \%)$ & $40(47.1 \%)$ & $35(38.5 \%)$ & $6(28.6 \%)$ & \\
\hline \multirow[t]{2}{*}{ LVSI } & Positive & $47(13.8 \%)$ & $21(19.8 \%)$ & \multirow[t]{2}{*}{0.163} & $34(13.6 \%)$ & $16(18.8 \%)$ & $13(14.3 \%)$ & $5(23.8 \%)$ & \multirow[t]{2}{*}{0.453} \\
\hline & Negative & $294(86.2 \%)$ & $85(80.2 \%)$ & & $216(86.4 \%)$ & $69(81.2 \%)$ & $78(85.7 \%)$ & $16(76.2 \%)$ & \\
\hline \multirow[t]{2}{*}{ LUSI } & Yes & $121(35.5 \%)$ & $50(47.2 \%)$ & \multirow[t]{2}{*}{0.039} & $94(37.6 \%)$ & $45(52.9 \%)$ & $27(29.7 \%)$ & $5(23.8 \%)$ & \multirow[t]{2}{*}{0.006} \\
\hline & No & $220(64.5 \%)$ & $56(52.8 \%)$ & & $156(62.4 \%)$ & $40(47.1 \%)$ & $64(70.3 \%)$ & $16(76.2 \%)$ & \\
\hline \multirow[t]{2}{*}{ CSI } & Yes & $26(7.6 \%)$ & $13(12.3 \%)$ & 0.167 & $20(8 \%)$ & $13(15.3 \%)$ & $6(6.6 \%)$ & $0(0 \%)$ & 0.063 \\
\hline & No & $315(92.4 \%)$ & $93(87.7 \%)$ & & $230(92.0 \%)$ & $72(84.7 \%)$ & $85(93.4 \%)$ & $21(100 \%)$ & \\
\hline FIGO 2009 & la & $172(50.4 \%)$ & $41(38.7 \%)$ & 0.048 & $139(55.6 \%)$ & $36(42.4 \%)$ & $33(36.3 \%)$ & $5(23.8 \%)$ & 0.000 \\
\hline & $\mathrm{lb}$ & $143(41.9 \%)$ & $51(48.1 \%)$ & & $91(36.4 \%)$ & $36(42.4 \%)$ & $52(57.1 \%)$ & $15(71.4 \%)$ & \\
\hline & II & $26(7.6 \%)$ & $14(13.2 \%)$ & & $20(8.0 \%)$ & $13(15.3 \%)$ & $6(6.6 \%)$ & $1(4.8 \%)$ & \\
\hline ESMO-ESGO- & Low risk & $108(31.7 \%)$ & $28(26.4 \%)$ & 0.367 & $88(35.2 \%)$ & $24(28.2 \%)$ & $20(22.0 \%)$ & $4(19.0 \%)$ & 0.088 \\
\hline classification & Intermediaterisk & $109(32.0 \%)$ & $31(29.2 \%)$ & & $71(28.4 \%)$ & $21(24.7 \%)$ & $38(41.8 \%)$ & $10(47.6 \%)$ & \\
\hline & $\begin{array}{l}\text { High- } \\
\text { intermediaterisk }\end{array}$ & $57(16.7 \%)$ & $18(17.0 \%)$ & & $42(16.8 \%)$ & 16(18.8\%) & $15(16.5 \%)$ & $2(9.5 \%)$ & \\
\hline & High risk & $67(19.6 \%)$ & $29(27.4 \%)$ & & $49(19.6 \%)$ & $24(28.2 \%)$ & 18(19.8\%) & $5(23.8 \%)$ & \\
\hline Radiotherapy & VRT alone & $238(69.8 \%)$ & $57(53.8 \%)$ & 0.003 & $178(71.2 \%)$ & $43(50.6 \%)$ & $60(65.9 \%)$ & $14(66.7 \%)$ & 0.007 \\
\hline & $\mathrm{EBRT} \pm \mathrm{VBT}$ & $103(30.2 \%)$ & $49(46.2 \%)$ & & $72(28.8 \%)$ & $42(49.4 \%)$ & $31(34.1 \%)$ & $7(33.3 \%)$ & \\
\hline Chemotherapy & Yes & $65(19.1 \%)$ & $30(28.3 \%)$ & 0.049 & $48(19.2 \%)$ & $25(29.4 \%)$ & 17(18.7\%) & $5(23.8 \%)$ & 0.217 \\
\hline & No & $276(80.9 \%)$ & $76(71.7 \%)$ & & $202(80.8 \%)$ & $60(70.6 \%)$ & $74(81.3 \%)$ & $16(76.2 \%)$ & \\
\hline Time interval & $\leq 49$ days & $244(71.6 \%)$ & $70(66.0 \%)$ & 0.277 & $180(72 \%)$ & $56(65.9 \%)$ & $64(70.3 \%)$ & $14(66.7 \%)$ & 0.736 \\
\hline and $\mathrm{R}$ & $>49$ days & $97(28.4 \%)$ & $36(34 \%)$ & & $70(28 \%)$ & $29(34.1 \%)$ & $27(29.7 \%)$ & $7(33.3 \%)$ & \\
\hline Overall & Yes & $13(3.8 \%)$ & $9(8.5 \%)$ & 0.069 & $6(2.4 \%)$ & $4(4.7 \%)$ & $7(7.7 \%)$ & $5(23.8 \%)$ & 0.000 \\
\hline & No & $328(96.2 \%)$ & $97(91.5 \%)$ & & $244(97.6 \%)$ & 81(95.3\%) & $84(92.3 \%)$ & $16(76.2 \%)$ & \\
\hline
\end{tabular}

$1=$ age $\leq 61$ years and $C A 125<37.7,2=$ age $\leq 61$ years and $C A 125>37.7,3=$ age $>61$ years and $C A 125<39.35$, and $4=$ age $>61$ years and CA125>39.35.LUSI=lower uterine segment invasion, LVSI=lymphatic vascular space invasion, EBRT= external beam radiotherapy, $\mathrm{VBT}=$ vaginal brachytherapy, $\mathrm{Ml}=$ myometrial invasion, $\mathrm{CSI}=$ cervical stromal invasion. $\mathrm{S}=$ surgery, $\mathrm{R}=$ radiotherapy

\section{Discussion}


At present, the commonly used clinicopathological risk stratification system does not include all used factors[6, 7]. According to the results of previous studies, preoperative tumor markers such as CA125 and age are prognostic factors for EC[8, 9]. In this study, the multivariate analysis of the 5-year OS of all patients with early-stage EC showed that none of the clinicopathological factors, except preoperative serum CA125 and age at diagnosis, had an independent prognostic advantage for OS. Therefore, for patients with early-stage EC, preoperative CA125 detection may help us distinguish between high- and low-risk groups earlier.

CA125 is a glycoprotein expressed in mesenchymal cells of the adult pleura, pericardium, and peritoneum, as well as cells derived from embryonic Mullerian tubes, namely, the oviduct, endometrium, and endometrial cells of the cervix. In premenopausal women, its level can be elevated due to a variety of physiological states and benign diseases, including inflammation, endometriosis, and menstruation, among others[10]. In EC, CA-125 levels have been shown to be associated with several clinicopathological factors[2-4, 8, 11-19]to predict LVSI, MI, lymph node metastasis, adnexal involvement, distant metastasis, OS, and DFS. There are different cutoff values for different age groups and for predicting different endpoints $[2,13,15,18]$. Therefore, CA125 is considered to be a valuable prognostic marker in EC patients.

However, most of the current studies on CA125 in EC are focused on finding ways to predict of advanced EC, such as distinguishing cases of extrauterine spread of EC from those with localized disease or monitoring advanced and recurrent cases[2-5, 11-15, 17, 18].Only two studies have reported the independent predictive value of CA125 for OS, LRFS and DFS after adjuvant therapy for early-stage EC[19, 20]. However, they were single-center retrospective studies with a small number of cases.

The purpose of this study was to evaluate the predictive value of CA125 for the prognosis of early-stage EC after surgery and adjuvant therapy. Due to the influence of age on CA125, the cutoff values of CA125 were different for different ages in this study. In the results, the CA125-positive group had a higher proportion of MI and LUSI, resulting in a higher proportion of patients with stage Ib and II disease, as well as a higher proportion of patients receiving EBRT and chemotherapy. OS was also significantly different between the two groups. Therefore, CA125 has its own value in the prognosis of early-stage EC. However, its AUC was only 0.590 (sensitivity=0.409, specificity=0.772). Therefore, the predictive value of CA125 alone is limited.

Age is another important clinical factor for EC. Our previous results showed that age was an important independent prognostic factor for early-stage EC and that the disease-related mortality of patients increased gradually with increasing age[9]. In this study, age was also shown to be an independent prognostic factor for early-stage EC. However, its AUC, sensitivity and specificity for EC were only $0.654,0.545$ and 0.765 , respectively.

Therefore, CA125 or age alone is of limited efficacy in predicting early-stage EC. When CA125 combined with age predicted the OS of earlystage EC, the AUC and sensitivity were significantly improved. In the previous literature, CA 125 combined with a variety of clinicopathological factors was also suggested to increase the accuracy of clinical diagnosis, such as MRI[21], Mesothelin[22] and HE4. At present, HE4 and CA125 are clinically approved tumor markers for the diagnosis and prognosis of EC. Among them, HE4 is considered to be more correlated with the prognostic factors of EC[5,23-26]. Its sensitivity to predict the survival of EC patientsis better than that of CA125 (0.71 vs.0.35), with no significant difference in specificity between the two markers. However, in the subgroup analysis, the sensitivity of HE4 in Asian patients (0.53) was significantly lower than that in Caucasian patients (0.86). Therefore, the role of HE4 needs further clinical investigation in Asian populations. In this study, OS was significantly different among the CA125-combined age groups with older age, and the CA125positive group had a worse prognosis. According to the current results, simple and easily obtained indicators can be used to distinguish the high-risk group of patients faster, which is quite helpful for decision-making in terms of the clinical treatment plan.

In addition, why did CA125 not show any statistical significance for DFS in this study? In most previous studies, CA125 elevation was associated with extrauterine invasion. However, in stage I-II EC, there is no extrauterine spread, such as lymph node metastasis or adnexal involvement. Therefore, CA125 did not show significance for DFS in these patients with early-stage EC in this study. However, the increase in CA125 indicates that there is a potential risk of tumor progression, such as MI and LUSI. Age plusCA125 as an overall indicator showed greater significance for OS. Therefore, for older patients with CA125 positivity, a more active treatment approach should be considered as the clinical treatment strategy, if possible.

This study is the only multicenter study to evaluate the prognostic value of preoperative serum CA125 for early-stage EC after initial treatment thus far. This study provides new and powerful evidence for the application of CA125 in early-stage EC. However, its limitation is that it is a retrospective study, which leads to the lack of some clinical information. In addition, due to the limitation of enrollment time, there is no molecular typing in the current data. Only patients with data on CA125 were included, which may result in selection bias.

\section{Conclusion}


Although preoperative CA125 has limited sensitivity in predicting the prognosis of early-stage EC after initial treatment, it remains a useful serum marker for the risk assessment of early-stage EC. Combining CA125 with age increases its predictive sensitivity. Thus, this study provides new and powerful evidence for the application of CA125 in early-stage EC.

\section{Declarations}

\section{Ethics approval and consent to participate}

This retrospective study was approved by the Ethics Review Committee of Peking Union Medical College Hospital, Chinese Academy of Medical Sciences [Protocol number S-K139]. The clinical trial ID of the study is ChiCTR-PRC-17010712. Evaluation of all data met the requirements of the Helsinki Declaration.

\section{Consent for publication}

The Authors declare that the paper is being submitted for consideration for publication in Radiation Oncology, that the content has not been published or submitted for publication elsewhere.

\section{Availability of data and material}

The datasets during and/or analysed during the current study available from the corresponding author on reasonable request.

\section{Competing interests}

The authors declare that they have no competing interests

\section{Funding}

The study is supported by the Nonprofit Central Research Institute Fund of Chinese Academy of Medical Sciences(No. 2019XK320046), grants from funds for Peking Union Medical College Postgraduate Education Reform Program (No. 10023201900104), and grants from the Ministry of Science and Technology of China (No. 2016YFC0105207).

\section{Authors' contributions}

Shuai Sun: Conceptualization; Data curation; Formal analysis; Investigation; Methodology; Resources; Software; Supervision; Validation; Visualization; Roles/Writing - original draft; Writing - review \& editing. Lijuan Zou: Data curation, Validation.Tiejun Wang: Data curation, Validation.Zi Liu: Data curation, Validation.Jianli He: Data curation, Validation.Xiaoge Sun: Data curation, Validation.Wei Zhong: Data curation, Validation.Fengju Zhao: Data curation, Validation.Xiaomei Li: Data curation, Validation.Sha Li: Data curation, Validation.Hong Zhu: Data curation, Validation.Zhanshu Ma: Data curation, Validation.Wenhui Wang: Data curation, Investigation, Resources.Meng Jin: Data curation, Investigation, Resources.Fuquan Zhang: Conceptualization; Data curation; Funding acquisition; Project administration; Resources; Validation; Writing - review \& editing.Xiaorong Hou: Conceptualization; Data curation; Formal analysis; Funding acquisition; Investigation; Methodology; Project administration; Resources; Software; Supervision; Validation; Visualization; Writing - review \& editing.Lichun Wei: Conceptualization; Data curation; Funding acquisition; Project administration; Resources; Validation; Writing - review \& editing.Ke Hu: Conceptualization; Data curation; Funding acquisition; Project administration; Resources; Validation; Writing - review \& editing.

All authors read and approved the final manuscript.

\section{Acknowledgements}

no

\section{References}

1. Engelsen IB, Akslen LA, Salvesen HB. Biologic markers in endometrial cancer treatment. APMIS: acta pathologica, microbiologica, et immunologica Scandinavica. 2009;117:693-707.

2. Chao A, Tang YH, Lai CH, Chang CJ, Chang SC, Wu TI, et al. Potential of an age-stratified CA125 cut-off value to improve the prognostic classification of patients with endometrial cancer. Gynecol Oncol. 2013;129:500-4.

3. Baser E, Gungor T, Togrul C, Turkoglu O, Celen S. Preoperative prediction of poor prognostic parameters and adjuvant treatment in women with pure endometrioid type endometrial cancer: what is the significance of tumor markers? Eur $\mathrm{J}$ Gynaecol Oncol. 
2014;35:513-8.

4. Chen L, Xiang Q. Effect of postoperative sequential chemoradiotherapy and concurrent chemoradiotherapy in treating advanced endometrial cancer. Journal of BUON: official journal of the Balkan Union of Oncology. 2020;25:2271-8.

5. Cymbaluk-Ploska A, Gargulinska P, Bulsa M, Kwiatkowski S, Chudecka-Glaz A, Michalczyk K. Can the Determination of HE4 and CA125 Markers Affect the Treatment of Patients with Endometrial Cancer? Diagnostics (Basel). 2021;11.

6. Concin N, Matias-Guiu X, Vergote I, Cibula D, Mirza MR, Marnitz S, et al. ESGO/ESTRO/ESP guidelines for the management of patients with endometrial carcinoma. Int J Gynecol Cancer. 2021;31:12-39.

7. Colombo N, Creutzberg C, Amant F, Bosse T, Gonzalez-Martin A, Ledermann J, et al. ESMO-ESGO-ESTRO Consensus Conference on Endometrial Cancer: diagnosis, treatment and follow-up. Ann Oncol. 2016;27:16-41.

8. Rizner TL. Discovery of biomarkers for endometrial cancer: current status and prospects. Expert Rev Mol Diagn. 2016;16:1315-36.

9. Hou XR, Yu L, Fau - Hu K, Hu K, Fau - Zhang F-Q, Zhang FQ. Outcomes of intermediate-risk to high-risk stage I endometrial cancer: 10year clinical experiences of using in-house multi-channel applicators in a single center. 2019.

10. Simmons AR, Baggerly K, Bast RC Jr. The emerging role of HE4 in the evaluation of epithelial ovarian and endometrial carcinomas. Oncology (Williston Park NY). 2013;27:548-56.

11. Myriokefalitaki E, Vorgias G, Vlahos G, Rodolakis A. Prognostic value of preoperative Ca125 and Tag72 serum levels and their correlation to disease relapse and survival in endometrial cancer. Arch Gynecol Obstet. 2015;292:647-54.

12. Chen YL, Huang CY, Chien TY, Huang SH, Wu CJ, Ho CM. Value of pre-operative serum CA125 level for prediction of prognosis in patients with endometrial cancer. Aust N Z J Obstet Gynaecol. 2011;51:397-402.

13. Pinar Cilesiz Goksedef B, Gorgen H, Baran SY, Api M, Cetin A. Preoperative serum CA 125 level as a predictor for metastasis and survival in endometrioid endometrial cancer. Journal of obstetrics and gynaecology Canada: JOGC = Journal d'obstetrique. et gynecologie du Canada : JOGC. 2011;33:844-50.

14. Lee JY, Jung DC, Park SH, Lim MC, Seo SS, Park SY, et al. Preoperative prediction model of lymph node metastasis in endometrial cancer. Int J Gynecol Cancer. 2010;20:1350-5.

15. Han SS, Lee SH, Kim DH, Kim JW, Park NH, Kang SB, et al. Evaluation of preoperative criteria used to predict lymph node metastasis in endometrial cancer. Acta obstetricia et gynecologica Scandinavica. 2010;89:168-74.

16. Kim HS, Park CY, Lee JM, Lee JK, Cho CH, Kim SM, et al. Evaluation of serum CA-125 levels for preoperative counseling in endometrioid endometrial cancer: a multi-center study. Gynecol Oncol. 2010;118:283-8.

17. Sebastianelli A, Renaud MC, Grégoire J, Roy M, Plante M. Preoperative. CA 125 tumour marker in endometrial cancer: correlation with advanced stage disease. Journal of obstetrics gynaecology Canada: JOGC = Journal d'obstetrique et gynecologie du Canada : JOGC. 2010;32:856-60.

18. Jiang T, Huang L, Zhang S. Preoperative serum CA125: a useful marker for surgical management of endometrial cancer. BMC Cancer. 2015;15:396.

19. Lin YJ, Hu YW, Twu NF, Liu YM. The role of adjuvant radiotherapy in stage I endometrial cancer: A single-institution outcome. Taiwan J Obstet Gynecol. 2019;58:604-9.

20. Yilmaz E, Gurocak S, Melekoglu R, Koleli I, Faydali S, Temelli O, et al. The Effect of Prognostic Factors and Adjuvant Radiotherapy on Survival in Patients with High-Grade Early-Stage Endometrial Cancer: A Retrospective Clinical Study. Medical science monitor: international medical journal of experimental clinical research. 2019;25:2811-8.

21. Tsikouras P, Koukouli Z, Bothou A, Manav B, latrakis G, Zervoudis S, et al. Preoperative assessment in endometrial cancer. Is triage for lymphadenectomy possible? Journal of BUON: official journal of the Balkan Union of Oncology. 2017;22:34-43.

22. Kakimoto S, Miyamoto M, Einama T, Matsuura H, Iwahashi $\mathrm{H}$, Ishibashi $\mathrm{H}$, et al. Co-Expression of Mesothelin and CA125 Is Associated with the Poor Prognosis of Endometrial Serous Carcinoma and Mixed Carcinomas Including Serous Carcinoma. Pathol Oncol Res. 2020;26:2299-306.

23. Zanotti L, Bignotti E, Calza S, Bandiera E, Ruggeri G, Galli C, et al. Human epididymis protein 4 as a serum marker for diagnosis of endometrial carcinoma and prediction of clinical outcome. Clinical chemistry laboratory medicine. 2012;50:2189-98.

24. Angioli R, Miranda A, Aloisi A, Montera R, Capriglione S, De Cicco Nardone C, et al. A critical review on HE4 performance in endometrial cancer: where are we now? Tumour biology: the journal of the International Society for Oncodevelopmental Biology Medicine. 2014;35:881-7.

25. Angioli R, Plotti F, Capriglione S, Scaletta G, Dugo N, Aloisi A, et al. Preoperative local staging of endometrial cancer: the challenge of imaging techniques and serum biomarkers. Arch Gynecol Obstet. 2016;294:1291-8. 
26. Li J, Wang X, Qu W, Wang J, Jiang SW. Comparison of serum human epididymis protein 4 and CA125 on endometrial cancer detection: A meta-analysis. Clin Chim Acta. 2019;488:215-20.

\section{Figures}

Patients receiving primary hysterectomy \pm bilateral salpingo-oophorectomy and adjuvant RT from 1999 to 2015 across 13 Chinese medical centers (1268)

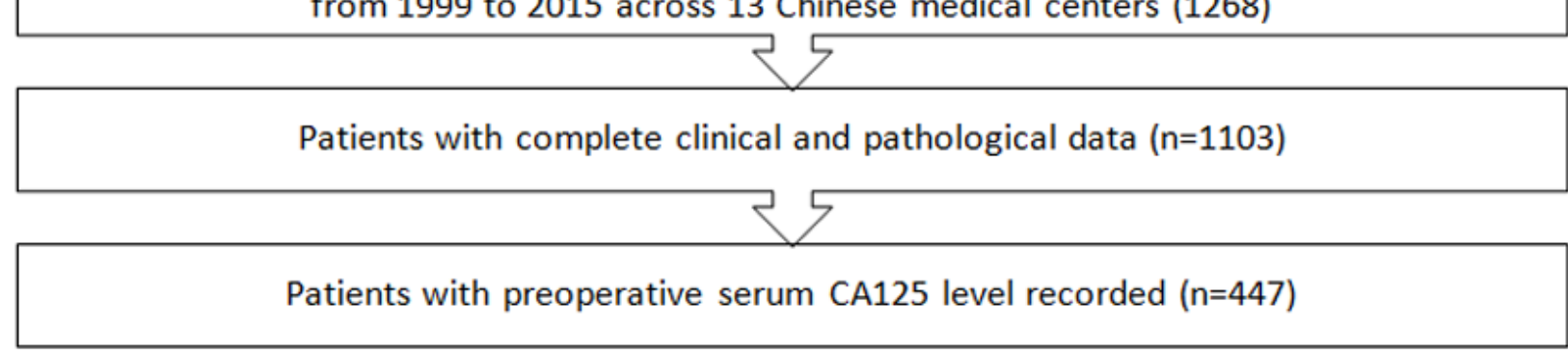

Figure 1

Flow chart of patient enrollment

Points
age
CA125positive
Total Points

\section{Figure 2}

Nomogram for 5y OS 


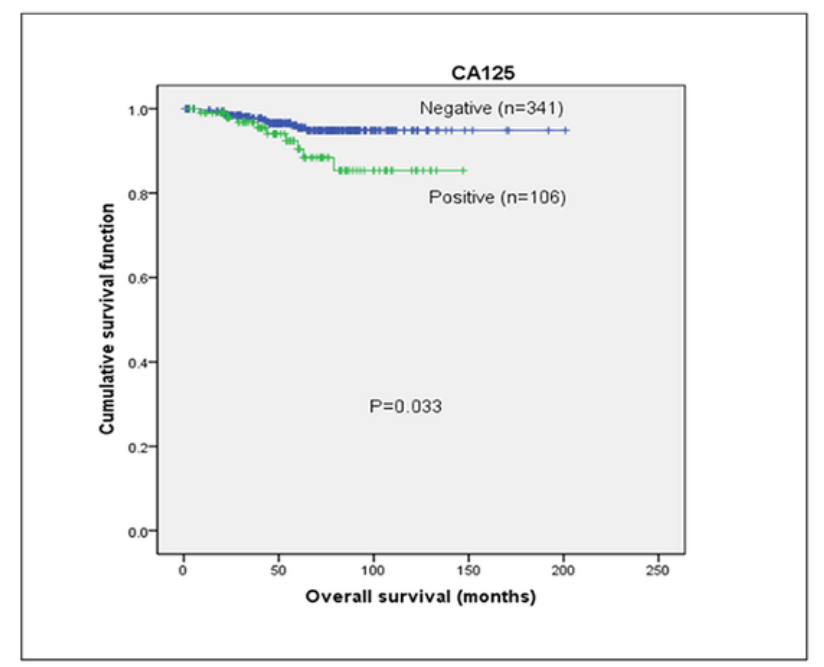

A

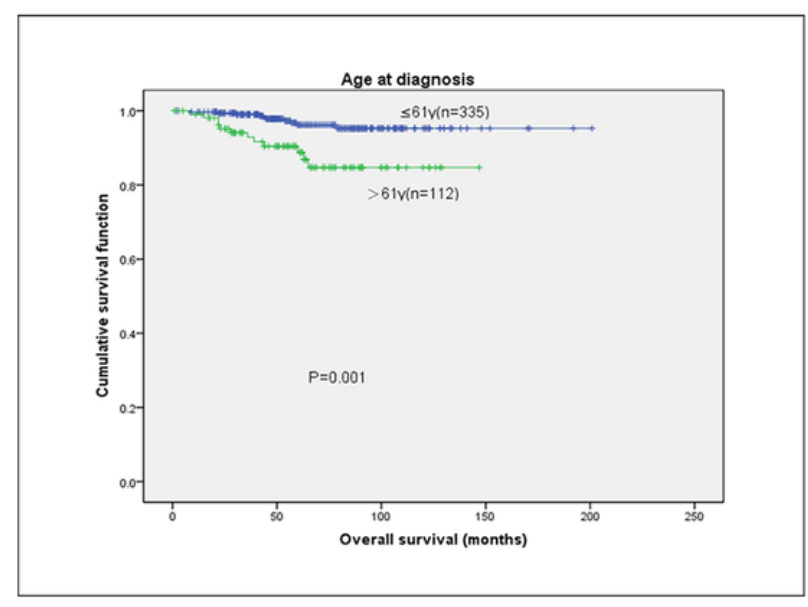

B

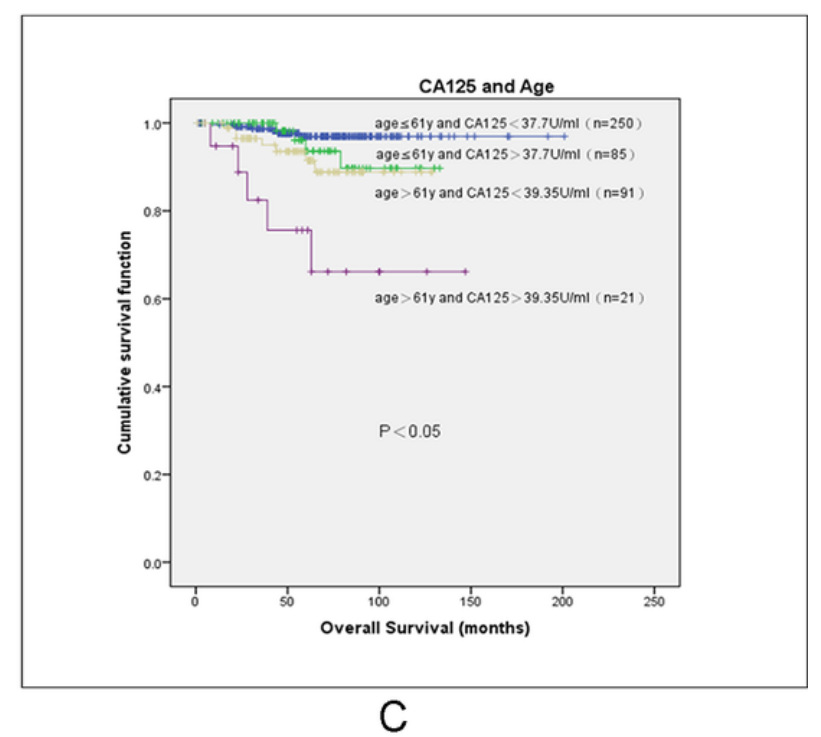

\section{Figure 3}

Survival function of CA125(3-a) and age(3-b) alone and in combination(3-c) to predict 5-year OS 